\title{
The Vulnerable Researcher Phenomenon
}

\author{
Xiaorong Tang1, Monit Cheung2, Shu Zhou², Patrick Leung' \\ ${ }^{1}$ Department of Social Work, South China Agricultural University, Guangzhou, China \\ ${ }^{2}$ Graduate College of Social Work, University of Houston, Houston, TX, USA \\ Email: 163sherry@163.com, mcheung@uh.edu,pleung@uh.edu
}

How to cite this paper: Tang, X., Cheung, M., Zhou, S., \& Leung, P. (2020). The Vulnerable Researcher Phenomenon. Open Journal of Philosophy, 10, 511-527. https://doi.org/10.4236/ojpp.2020.104036

Received: August 11, 2020

Accepted: November 17, 2020

Published: November 20, 2020

Copyright (c) 2020 by author(s) and Scientific Research Publishing Inc. This work is licensed under the Creative Commons Attribution International License (CC BY 4.0).

http://creativecommons.org/licenses/by/4.0/ (c) (i) Open Access

\begin{abstract}
Purpose: The purpose of this study is to address challenging issues in protecting researchers in risky situations. Method: A case study addressed the importance of risk prevention and researcher safety. In six interviews, a social scientist shared feelings of vulnerability when she conducted ethnographic research with sex workers and gang members. Results: Qualitative results explain the concerns from the eyes of a vulnerable researcher in six factors: Support, Safety, System Connection, Situations, and Struggles. The vulnerable researcher phenomenon is defined as the emotional labor faced by the researcher in situations that are dangerous and risky. Implications: While taking care of the research subjects' well-being, researchers must also be reminded that ethical behaviors in their field also include self-protection. Social scientists must advocate for a standard protocol in IRB applications to include protecting researcher safety and ensuring identity privacy.
\end{abstract}

\section{Keywords}

Researcher Vulnerability, Researcher Protection, High-Risk Research, Institutional Review Board (IRB), Research Ethics, Self-Disclosure, Researcher Anonymity, Case Study

\section{Introduction}

Research has provided support for constant policy examination of the Institutional Review Board (IRB) or research ethics committee to support its various functions and define the concept of vulnerability for the protection of human subjects (Lajoie et al., 2020; Noue \& Bush, 2010). However, limited studies have described what guidelines IRBs could provide for the protection of researchers who plan to collect data in the field, particularly in high-risk situations (Kästner et al., 2015). As social research has a strong focus on confronting social injustice and working with marginalized populations, many researchers have encountered 
highly sensitive debates which might place them in dangerous or uncomfortable situations during their data collection processes because of their field work environment (Muller \& Gubrium, 2016). In the field of anthropology, Behar (1996) coined her theory of the vulnerable observer. Behar (1996) advised researchers, before stepping into ethnographic research, to take care of themselves first and understand the impact of detachment on their emotional responses to the observations. It is also important for field researchers to design their safety protection plan as part of the research plan. Researchers must also be aware that the emotional labor and vulnerability that they may encounter in the field is essential to working with these populations. Therefore, their pre-research work should include carefully prepared justice-oriented questions to ask the subjects. This article starts with an analysis of the principles of human subject protection as a foundation for exploring ways to define and reduce the vulnerability experienced by researchers who feel unsafe and/or emotionally taxed when working in the field.

\section{Non-Maleficence Is a Core Value}

The core value in the process of protecting human subjects is "doing no harm and getting it right" (Hernández et al., 2013: p. 43). This value has become the cornerstone for supporting three principles in bioethics. These principles are essential for affirming the function of research ethics in protecting researchers.

\subsection{Do No Harm}

As the most important precept in research ethics, primum non nocere (first, do no harm) illustrates the importance of preventing vulnerability faced by research subjects using the principles of cognitive, juridic, deferential, medical, allocational, and infrastructural protection strategies (Kipnis, 2001). These six areas of protection emphasize the use of a concrete plan to protect research subjects from potential danger so that their participation will not negatively impact their functioning, abilities, and status. Literature has addressed how to protect "vulnerable populations" (Yoshikawa et al., 2017: p. 459) such as the LGBTQ community, people with impaired abilities, subjects with emotional instabilities or challenges, clients with HIV/AIDS, drug users, people in residential care, children, refugees, migrant women, orphans, and prisoners (Cheney, 2011; Hernández et al., 2013; Merry et al., 2011; Ryan et al., 2019; Wakai et al., 2009). When considering the vulnerability of persons within research, the focus is consistently placed on the research subjects and not the researchers themselves. Recently, this has been presented as an issue for ethnographic researchers as advocated by Taylor (2019) who studied violence and trauma in the field.

\subsection{Privacy and Confidentiality}

Second, the do no harm principle aims to protect the right of privacy and confidentiality of the subjects and eliminate any safety or change-of-status concerns 
from participation or nonparticipation, particularly with traumatized patients (Etherington, 2007). Although this work-ethics principle governs researchers' duties and protects subjects' identity, it does not include methods to mask the researcher's name or affiliation to protect the researchers from harm (Leigh, 2015). Since researchers' names can be disclosed through the research consent and publication process, they have not been offered the legal right to be an anonymous researcher. Researcher's risks as a professional are further discussed in Brougham \& Uttley's (2017) study in correctional settings.

\subsection{Not to Risk}

Third, the better to not do something than to risk principle aims to avoid doing more harm than good as a consequence of subject participation. The use of exclusion criteria and no-risk assessment must be integrated into participant selection to lower any potential participation risk (see Benoit et al., 2012 for discussion on trust, engagement, and protection). Additionally, follow-up counseling services for the subjects must be available in the case of unforeseen needs or emotional reactions (Boothroyd \& Best, 2003; Delva, 2007). These possible prevention and intervention measures illustrate a dilemma between completing a study and ethically exercising the "duty of care" principle when sensitive data collection is viewed as a risky process (Bahn \& Weatherill, 2013: p. 19). While this is a well-intended principle, it only protects the well-being of research subjects while the welfare of the researchers may be neglected.

\section{Principles of Researcher Protection}

Literature tends to focus on research skills when discussing sensitive topics with subjects such as organized criminals (Lambrechts, 2014) or sex workers (Horn, 2007). The human protection strategies are mainly respondent-focused; for example, they include suggestions on how to present sensitive questions to avoid overwhelming the respondents and remain neutral and nonjudgmental to enhance participation (Bourne \& Robson, 2015). However, recommendations on how to minimize the psychological impact and potential physical harm that these field research topics can have for the researchers have yet to be explored. The researchers may discover that demonstrating empathy and exploring difficult topics with subjects can be exhausting. Although researchers may develop a protective plan for themselves if the study requires them to enter a dangerous or emotionally provoking environment, little guidance has been provided on the creation of such a plan in all research projects, especially when the safety threat is perceived or experienced only after the start of data collection.

\section{Purpose of This Study}

In this study, the purpose of the interviews was to provide methodological guidelines for teaching purposes and to document how a researcher handled her research procedures to protect human subjects in a dangerous environment or on a sensitive topic. Based on a series of interviews with a social scientist ("scho- 
lar") whose work focused on the health status of gang members and sex workers, we found six themes from the scientist's first-person experience that was associated with researcher vulnerability. Even though the data collected for this study was from one scholar's experience, it depicted the importance of researcher protection.

The first step of this study was to seek a definition of researcher safety. Even though few studies addressed safety from the researcher's perspective, Emerald \& Carpenter (2015) found that field-based research can cause "emotional labor" to the researcher, meaning that there is intensive work toward the data collection processes that demand time and effort to overcome barriers in dangerous or emotionally intensive situations. With this definition, we interviewed a scholar whose research was connected with vulnerable populations. The purpose was to document how she protected herself during high-risk research situations.

\section{Method}

A series of six semi-structured interviews were conducted with a scholar who has spent extensive time conducting participatory research, studying topics related to sex workers and gang members. Two interviewers used a set of guided questions which focused on the principles for conducting sensitive research with attention to confidentiality, anonymity, values, ethics, commitment, and risk factors in this participatory research. This research was approved by the IRB of the second author's affiliated university. The third author video-recorded the entire process of the interviews until all guided questions were asked and answered. She also transcribed the contents for the team to read individually. Transcription was then completed with two researchers' inputs, who spoke the same language as the scholar, and validated the contents with back-translation. The entire team analyzed the major themes extracted from the transcripts and focused on how the scholar protected her safety and prevented any forms of researcher vulnerability.

\section{Data Collection}

For data collection purposes, the term scholar was used to represent the interviewee who anonymously and voluntarily agreed to provide data for this study. The term researchers (or we) was used to represent the interviewers who were present in the interview process, including the coders. The scholar, who had expertise in research on sensitive topics, was invited and interviewed by the researchers to identify major challenges in her research studies.

- A total of six face-to-face semi-structured interviews were scheduled with the scholar to describe: 1) the recruitment process of sex workers and gang members as research subjects; 2) the definition of sensitive topics; 3) feelings and reactions to the subjects' responses; 4) debriefing of the researcher's vulnerability; and 5) self-care during and after each research project.

- The interviews were open-ended, discovery-oriented, and well suited to probe for descriptions and interpretations of personal experiences (Crabtree \& Mil- 
ler, 1999). This method is recommended to encourage the scholar to express life experiences related to her study topics, and how she viewed herself during the research process (Horstman et al., 2008; Matsudaira, 2003). Since the interview questions were intended to stimulate personal and emotional responses, we planned six interviews so that the scholar would have sufficient time to answer.

- During these interviews, the scholar answered a set of questions with prompts and follow-up questions related to her studies with sex workers and gang members as subjects (see Table 1). These questions were categorized into seven areas: Topic Selection, Decision Making, Role as a Researcher, Sampling, Values and Ethics, Challenges, and Recommendations. Based on the interview transcripts, the research team analyzed how the scholar processed her decision to enter a research site and what methods were used for her selfprotection.

- With a focus on vulnerable research circumstances, we used coding methods to consolidate the data into themes for researcher protection and ordered it in terms of importance as perceived by the scholar. The scholar then reviewed the themes generated from her interviews and provided additional feedback, which focused on potential researchers' risks in hindsight, to validate the analyses presented in this article.

Table 1. Probing questions for the interviews.

1) How did you determine your research topics?

2) What motivated you to choose a topic of high sensitivity?

3) When you decided on your study topic, did you face any challenges in finalizing your research questions?

4) When your topic and research questions were finalized, what support or resources did you need to carry out the research?

5) How did you determine which research site(s) to implement your study?

6) When you first entered the research site, what role did you play?

7) How did you explain your researcher role to the participants?

8) How did you engage the participants in your introductory meeting with them?

9) How did you build rapport and trust with the participants?

10) How did you expand the pool of your subjects?

11) How did you recruit and invite your subjects to participate in your study? Did you provide any incentive? If so, how did your subjects view the incentive?

12) How do you handle confidentiality and anonymity of your subjects?

13) Did any of the data present conflict with your personal or professional values?

14) How did you resolve your conflict? How did you protect yourself from harm (from the conflict or in the situation)?

15) How did you present your findings without disclosing your research location or the subjects involved?

16) When you sensed that someone needed counseling or other social services, what procedures did you use to safeguard confidentiality and yet make appropriate referrals?

17) How would you be sure that the government would not check with you about your subjects?

18) With whom could you debrief your feelings, particularly emotional ones?

19) What was the biggest challenge during each of your research processes?

20) Any differences between the two types of research?

21) Was the researcher's gender an important consideration, particularly when studying sex workers?

22) Did you experience any role conflict in the process of data collection? 


\section{Results}

The main content from the interviews compares how interviewing sex workers and gang members were different. This scholar used examples to illustrate how she entered the research sites and described the challenging situations arising from her studies. The research team finalized these themes and named them Vulnerable Researcher Factors: Support, Safety, System Connection, Situations, and Struggles. While we recognize the limitation of generating findings from a case study, these factors are described with the scholar's direct quotes to promote awareness of the vulnerable researcher phenomenon and its challenges.

\subsection{Support}

Beyond being a keen researcher who wanted to conduct research that was seldom studied, the scholar felt that she had support that allowed her to continue working towards her research goal. The first theme, support, refers to a motivating source for continuously working with vulnerable populations. Such support comes from home, work, and the informants. Without sufficient support, the scholar stated that she would not have designed, implemented, or completed her studies.

I had two motivations to choose a high sensitivity topic. 1) I like adventures and challenges. The more sensitive the topic is, the more difficult the research will be. 2) Geographical convenience, good timing, and human relations. I happened to know someone who can introduce me to my target population.

After I finalized my research questions, I needed a lot of support in different areas. First, academic support from colleagues, classmates, professors, and other professional friends. Second, life support from my family. For example, my husband helped to take care of our kids when I was out for research. Third, psychological support. For instance, my husband supported me to research these special topics, and my informant agreed with me about researching the rural culture.

As an important element to conducting high-risk research, support helps strengthen the researcher's courage to enter the field and prepare a research path. Along with personal commitment, the cycle of support continues. Once in the field, the scholar establishes personal resiliency by trusting her commitment toward personal fulfillment.

Fortunately, I was able to debrief my feelings to my support network, especially when I was being emotional. For example, colleagues, husband, friends, classmates, professors, and other professional friends.

\subsection{Safety}

The second factor, safety, refers to the perception that self-protection is rooted in an awareness of security precautions. This factor is closely connected to the 
support factor. Although the scholar first felt supported, she must also deal with the reservations expressed by her family, mainly due to their concern about her safety.

I had to give up gathering further information because of my family's values [and] to resolve the conflict. I could have conducted further research about the gangs. I didn't continue because I felt it would have been dangerous. I guess that [i.e., learning about the risk before starting] was how I protected myself from harm.

From the scholar's perspective, the safety concern was not her priority until she started working in a high-risk environment. Although she has a strong sense of safety alert, she came back from her research feeling unsafe. In hindsight, the scholar felt that training would have helped her check-in with her sense of vulnerability before getting started with her projects.

Probably I placed myself at risk. On one occasion, two gangs started fighting when I was eating between them. I knew I was counted as a witness because I was there. However, I didn't know much what was going on at that time and felt clueless.

\subsection{System Connection}

The scholar found it helpful to be acquainted with trusted members of the target population in any research study. The use of her system connection method helped her learn about the research environment and deal with adversities when she enters research sites. Her informants not only served as her proof of being a trustworthy researcher, but also a bridge to recruit subjects who typically would not want to be studied without a mutual connection.

I happened to know an informant from that village, so it was a natural decision to choose that village for my research... I didn't decide to research them until my informant described more about their lives.

Regarding the gang research, my informant told me that his village had played a key role in a gang and described the gang's lifestyle.

She stated that showing her sincere attitude to the community allowed her to know several informants who guided her into reaching the research population.

I mainly used my informants as the central point to build rapport and trust with the participants. My informants brought me to their lives to listen, see, and observe. After I became more familiar with some of the sex workers or their families, I actively talked with them to deepen our relationship, finding common topics, and discover their needs.

However, the assistance from the connection system also led her into a vulnerable position because these informants would leave her after the initial introduction. 


\subsection{Situations}

The scholar described how she explained her multiple roles to her subjects in a language that was easy to understand.

It took me some time to explain my role to the participants and others involved. When I was conducting the gang research, I told my informants that I need to write a master thesis but they didn't understand. They understood after I explained that it was an assignment about observing people's living conditions. When we were with other research subjects, my informants introduced me as a master student from Shanghai. Sometimes, they didn't formally introduce me and I was just one of their acquaintances. When I was conducting the sex worker research, I told my main informants that I researched the rural culture. They were supportive. Other times, my role as an acquaintance of a research subject or a guest placed me there as an active listener.

When I first entered the research site, my role was different depending on who I talk to. I was a researcher when I was with my informant, and I was a professor or a guest when I was with other researchers. I also played different roles when I was with different people. For example, I became a friend's relative and professor when going out with my informant.

As the research subjects understood why the scholar was observing and asking questions, they were able to share different events and related situations that they faced. The scholar described her various roles in her studies: a researcher in the field to collect sensitive data, a professor with the mission of gaining knowledge, and a social scientist caring about the interviewees' well-being. She addressed her vulnerability as a reaction to the fear of not protecting her safety, a concern that research questions were not fully understood by the subjects, and an extensive feeling about the various situations she and her subjects faced during the interviews.

\subsection{Struggles}

The scholar described struggles as a vulnerability factor as she must resolve her personal and professional value conflicts. When working with vulnerable populations, the researcher must be aware of how personal boundaries could be crossed, particularly during the information gathering stage. As a researcher who was respected in the community she worked with, the scholar felt uncomfortable sharing the focus with other uninvolved individuals, particularly her family, because they might present concerns or biases that could become a personal struggle for the researcher to deal with.

When a sex worker came to the Province, my family did not allow me to meet her. As a result, I had to give up further contact with her. My professional values told me that I should help the sex workers but [I must] limit my contact with them. 
However, not disclosing the research focus might also be a conflict with the scholar's value of informing her family of her struggles. On one hand, keeping one's research topic confidential might be a core value for conducting highly sensitive research. On the other hand, not being able to share that topic could leave the researcher feeling unsupported. Her struggle was connected to her professional commitment to helping these subjects. As a member of a helping profession, the researcher would value offering help over data collecting.

I experienced some role conflicts when I was collecting my data. For example, conflicts between the role of a researcher and a friend. When I was observing research subjects or talking with them, they treated me like a friend or a researcher at best. However, I knew that I was researching a group of marginalized populations.

This type of struggle was illustrated with her tendency to lend a helping hand during the studies. With both examples of sex worker subjects and gang study subjects, the scholar illustrated that being clear on defining the researcher's role and boundary was of utmost importance in preventing personal struggles.

\section{Discussion}

In this study, researcher vulnerability was addressed with an emphasis on protecting researcher privacy. The scholar suggested a definition of researcher vulnerability to explain her feelings of being a vulnerable researcher. Generated from the interviews and literature on researcher vulnerability, the data show that there is not an all-encompassing definition to describe how the scholar knew what risk or harm to anticipate, what protection should be required in different situations, and how the perceived vulnerability could be described in concrete terms. Table 2 outlines the principles of protecting human subjects and reducing researcher vulnerability from the scholar's perspective. The scholar's use of terms such as risk, harm, potential danger, and vulnerability was connected to her perception of the situations she encountered; it was not a result of actual harm she experienced. In these interviews, she indicated six areas of concern:

Table 2. Principles for protecting human subjects vs. reducing researcher vulnerability.

\begin{tabular}{|c|c|c|}
\hline Comparing... & Principles of Human Subjects Protection & $\begin{array}{l}\text { Principles of Vulnerable } \\
\text { Researcher Protection }\end{array}$ \\
\hline Differences & $\begin{array}{l}\text { - Higher priority and more attention } \\
\text { - Existing principles: cognitive vulnerability, } \\
\text { juridic vulnerability, deferential } \\
\text { vulnerability, allocational vulnerability, and } \\
\text { intrastructural vulnerability } \\
\text { - Researchers' responsibilities to protect } \\
\text { human subjects are highlighted and required }\end{array}$ & $\begin{array}{l}\text { - Lower priority and less attention } \\
\text { - No principles specified for IRB } \\
\text { - Institutional accountability to } \\
\text { protect the well-being of } \\
\text { researchers are not addressed }\end{array}$ \\
\hline Similarities & $\begin{array}{l}\text { - Safety protection and ethical commitment for } \\
\text { - Both subjects and researchers face safety chal } \\
\text { to advocating for social justice } \\
\text { - Safety concern is connected with one's feeling }\end{array}$ & $\begin{array}{l}\text { "duty of care" } \\
\text { llenges when the studies are related } \\
\text { g of vulnerability }\end{array}$ \\
\hline
\end{tabular}




\subsection{Researchers' Readiness}

Irrespective of whether the topic is a highly sensitive issue, the scholar felt it is the researcher's responsibility to create a detailed plan to protect human subjects and to address the risk of subject vulnerability (similar to the studies engaging the subjects as partners described in Kia-Keating et al., 2017). To minimize subjects' risk, particularly in participatory research, the researcher agreed that it was important to attend to self-care and self-protection practices (Dickson-Swift et al., 2006; 2008; 2009; Wilson et al., 2018). It is essential that a participatory researcher must perceive self-protection as a required professional behavior when planning a sensitive field research. In particular, Dickson-Swift et al.'s (2008) article addresses risk assessment for researchers, discusses strategies for their management, and summarizes the guidelines developed by the Social Research Association (SRA) in the United Kingdom called "A Code of Practice for the Safety of Social Researchers" for assessing and mitigating risk to researchers.

\subsection{Ethical Challenges}

Kelleya et al. (2016) addressed the ethical challenges as an important step before planning a research study with vulnerable subjects such as orphans. With their research experiences in seven countries, they found it difficult to obtain guardian consent for the subjects particularly related to risk assessment among the orphans with multiple challenges. Therefore, they recommended training for these guardians or caregivers, but they did not provide suggestions on how to protect the researchers when these subjects challenged them about their study targets. When the researchers could obtain consent from the caregivers, they found that these caregivers had limited knowledge about the orphans or their mental health conditions. How do researchers determine who could be the best informants on behalf of these children?

\subsection{Researchers' Emotional Vulnerability}

The scholar had performed intensive activities that could cause physical and emotional exhaustion. In the literature, when facing dangerous situations or listening to highly emotional intense narratives, social researchers must attend to their emotions and the demanding tasks that are required for successful study completion (Yiu, 2015). According to the scholar, vulnerability is defined based on the situation a person is in. This concept is also known as contextual vulnerability, in which researchers are typically "overwhelmed by [the] context" in which they conducted an intensive data collection process (Duncan \& Stephenson, 2013: p. 556). This type of vulnerability arises when the researchers are not prepared to deal with the emotional or physical intensity of the study's context. The scholar felt that if researchers failed to address their vulnerability before the start of the study, they may be compromising their well-being (Mitchell \& Irvine, 2008). What could researchers do to reduce their emotional vulnerability when selecting a human subject protection plan? 


\subsection{Researchers' Safety and Well-Being}

The scholar disclosed that although she did not always feel safe when interviewing her subjects for her study, she did not stop the data collection process as it is part of her commitment as a social scientist. All health professionals have the responsibility to treat clients and colleagues with respect and protect them from harm. While healthcare professionals must be knowledgeable about research practices, researcher safety is rarely addressed. Parker \& O'Reilly (2013) discovered through their dyadic interviews with a research subject that threat to safety was not only experienced by the on-site researcher, but also the transcriptionist who was not on the site but listened to all the emotionally taxing conversations. Such a threat could be prevented if careful planning would include suggestions for immediate responses in threatening situations.

In a commissioned inquiry project, a group of researchers in Wales (Bloor et al., 2007) recommended for researcher protection principles to be included in an institutional risk management plan to protect researchers' physical safety and emotional well-being.

\subsection{Methodological Issues}

The scholar's experiences as a social researcher, case vignettes, story-sharing, and ethnographic studies have been used to illustrate the lives of vulnerable populations. The question is, have researchers learned specific methods for researching highly sensitive topics? As participatory research is commonly used to reach vulnerable populations, living or working within this community can put researchers in a vulnerable position. Horn (2007) cautions researchers who choose methods that are either too broad or too narrow as they are likely to receive negative attention from the subjects. For example, researchers who provide self-disclosure because their subjects inquire about it may receive negative responses. In some community-based studies, their research participants have acted as "coresearchers" to determine which information can be included in the study (Ismail et al., 2014). Research on how these participatory research methods can lead to researcher vulnerability is still lacking. For example, Kaplowitz (2000) found that individual interviews can be 18 times more likely to generate socially sensitive discussions than focus groups, but the possibility that researchers can be at risk when exploring such topics in that setting was not explored. Bourne \& Robson (2015) found that to enhance participation in studies investigating sexual behaviors, it is essential for researchers to be neutral and nonjudgmental. Based on this scholar's experience, researcher vulnerability must be explored.

\subsection{Researcher Anonymity}

The scholar requested to remain anonymous for this study. This request illustrates how researcher vulnerability occurs not only during the data collection process or when presenting study outcomes, but continues in the future as the researcher's identity has been disclosed through professional activities such as 
presentations and publications. Although some researchers collect highly sensitive information from their subjects, there is no mandate to protect the researchers' identities. As a result, researchers become vulnerable to negative and provocative attacks during data collection (Benson, 2019; Dickson-Swift et al., 2006, 2009). Researchers' identities are easily traced when they publish the results of their study, leading to a high level of personal vulnerability. Since researcher identity and professional affiliation provide proof of credibility, researchers typically do not object to disclose their identity, even when the subject matters in their report are secretive or highly sensitive. The researchers are placed in a difficult situation because associations between them and their studies increase the potential risk they may face after their studies are published.

\section{Practical Implications}

This study provides implications for research and education on research ethics. The interview dialogue from the scholar has demonstrated two major characteristics of the vulnerable researcher. First, the vulnerable researcher is not only a person, but also a public figure who displayed self-awareness and resilience in conducting observational or participatory research. Second, the vulnerable researcher is a protector who safeguards others but not themselves. It is expected that these researchers are highly motivated and understand how to protect themselves and others, access data with respect, know how to deal with crises and conflicts, and maintain a clear professional boundary. However, they are aware of the importance of being protected physically, personally, and emotionally, with a procedure that allows for researcher anonymity when reporting highrisk results. Additionally, they can provide examples of how to use appropriate channels to process their vulnerability in various stages of their research-before, during, and after the data collection process. Being a public figure and a protector, researchers bear the responsibility to protect their research subjects and they must also express their need for researcher protection.

\subsection{Formal Definition of Researcher Vulnerability}

In this case study, the scholar did not offer a formal definition of researcher vulnerability. Nevertheless, she viewed her vulnerability from a positive perspective in that she finally completed her time-intensive studies without being physically harmed. Her research process provides a learned lesson for social scientists to emphasize educational training on how to protect researchers. This suggestion also provides support to deliver IRB training with positivity, i.e., addressing the need and importance of promoting researcher protection.

In terms of conceptualizing the phenomenon of researcher vulnerability, the qualitative data support this phenomenon as a reminder that a research protocol must be developed for protecting the researchers. Researcher protection is the process involved to ensure the implementation of a high-quality protection scheme that is designed to overcome vulnerability on both ends-the researchers 
and the research subjects. Since these two concepts have not been clearly defined in health and mental health studies, additional research must be conducted to identify key elements in developing researcher protection guidelines or IRB protocol that contains safety plans for the researcher. These specific IRB procedures can accomplish this protection by developing clear research prototypes before researchers are engaged in unpredictable situations. Thus, the researchers can practice protection strategies to avoid threats to their emotional and physical safety.

\subsection{Strength of This Study}

The strength of this study is it provided a first-hand information from the angle of a researcher with in-depth experience in conducting studies with gang members and sex workers. Through multiple interviews, valuable insights were obtained, such as how the scholar needed support to process the aftermath of feeling vulnerable but could not share with her family due to confidentiality, and how her family's negative judgment of her work may affect her future studies with high-risk populations. A major limitation of this study is that it only had one case study to illustrate the importance of the vulnerable researcher phenomenon; the dialogue provided in this study was limited to a scholar's experience with two high-risk populations-gangs and sex workers. Nonetheless, this result provides a beginning step for researchers to be more aware of their research surroundings. These include how their thinking may influence the subjects' answers and how the researchers may be negatively perceived by potential subjects (Snoek \& Horstkötter, 2018).

\subsection{For Future Research}

The six themes identified to be vulnerable researcher factors can be used to initiate a dialogue on researcher protection in health research. This dialogue should pay attention to the multiple roles of being a researcher, educator, and practitioner. These findings also outline the major principles for researching high-risk topics, including awareness of one's vulnerability, prevention, collaboration, advanced training on research, and practice ethics. In addition to proposing IRB guidelines that include protecting researchers in ethnographic and other field studies in health research, the scholar also recommended universities to form a counseling committee to help researchers process their emotional reactions to research findings and debrief on high-risk encounters during their data collection process. Her insights into helping the vulnerable researcher bring forth an idea similar to self-care in health services: all practitioners, including researchers, should have a chance to process intense emotions generated from their practice to enhance healthy continuation of their work.

\section{Conclusion}

The purpose of this study is to address challenging issues in protecting re- 
searchers in risky situations and report the discovery of the vulnerable researcher phenomenon through a case study on gang members and sex workers research. Vulnerability is reciprocal when research subjects sense the risk of participating in research while their researchers also feel the negative impact of a risky environment. Researchers are meticulously trained to protect the safety and identity of research subjects and prevent potential risks associated with the research. Guidelines have been developed to protect human subjects, with a specific focus on the vulnerable populations (see the most recent guidelines in von Benzon \& van Blerk, 2017). When Peterson et al. (2017) proposed a peer-led model to educate researchers on how to deal with vulnerable situations, they mentioned the importance of promoting researchers' health. However, there is currently no specific protocol that addresses researchers' mental health or concrete guidelines that aim to protect researchers' privacy.

In this case study, six vulnerability factors were identified in the scholar's description of her research experience. The research protocol should include these factors as part of the researchers' self-care plan. When self-disclosing one's multiple roles as a researcher, the vulnerable researcher must also answer this question on self-protection: "What can researchers do to protect themselves when and after conducting research and publishing results that may put them at risk?” Future topics of exploration on this subject may include how to increase institutional involvement in researcher protection to reduce researcher vulnerability, how to train researchers to better protect themselves, and what role the IRB could play in the protection of vulnerable researchers. Furthermore, more research should be conducted to test existing risk-management protocols developed by organizations that routinely operate in high-risk environments. A researcher protection plan is the next priority step.

\section{Conflicts of Interest}

The authors declare no conflicts of interest regarding the publication of this paper.

\section{References}

Bahn, S., \& Weatherill, P. (2013). Qualitative Social Research: A Risky Business When It Comes to Collecting “Sensitive” Data. Qualitative Research, 13, 19-35. https://doi.org/10.1177/1468794112439016

Behar, R. (1996). The Vulnerable Observer: Anthropology That Breaks Your Heart. Boston, MA: Beacon Press.

Benoit, E., Pass, M., Randolph, D., Murray, D., \& Downing, M. J. (2012). Reaching and Engaging Non-Gay Identified, Non-Disclosing Black Men Who Have Sex with Both Men and Women. Culture, Health \& Sexuality, 14, 975-990. https://doi.org/10.1080/13691058.2012.709640

Benson, M. (2019). On Goffman: Ethnography and the Ethics of Care. The Sociological Review.

https://www.thesociologicalreview.com/on-goffman-ethnography-and-the-ethics-of-care/

Bloor, M., Fincham, B., \& Sampson, H. (2007). Qualiti (NCRM) Commissioned Inquiry 
into the Risk to Well-Being of Researchers in Qualitative Researcher. http://eprints.ncrm.ac.uk/407/1/CIReport.pdf

Boothroyd, R. A., \& Best, K. A. (2003). Emotional Reactions to Research Participation and the Relationship to Understanding of Informed Consent Disclosure. Social Work Research, 27, 242-251. https://doi.org/10.1093/swr/27.4.242

Bourne, A. H., \& Robson, M. A. (2015). Participants' Reflections on Being Interviewed about Risk and Sexual Behaviour: Implications for Collection of Qualitative Data on Sensitive Topics. International Journal of Social Research Methodology, 18, 105-116. https://doi.org/10.1080/13645579.2013.860747

Brougham, P. L., \& Uttley, C. M. (2017). Risk for Researchers Studying Social Deviance or Criminal Behavior. Social Sciences, 6, 130. https://doi.org/10.3390/socsci6040130

Cheney, K. E. (2011). Children as Ethnographers: Reflections on the Importance of Participatory Research in Assessing Orphans' Needs. Childhood, 18, 166-179. https://doi.org/10.1177/0907568210390054

Crabtree, B. F., \& Miller, W. L. (1999). Doing Qualitative Research (2nd ed.). Thousand Oaks, CA: Sage.

Delva, J. (2007). The Human Subjects Protection Process: A Subjective View. Social Work, 52, 101-102. https://doi.org/10.1093/sw/52.2.101

Dickson-Swift, V., James, E. L., Kippen, S., \& Liamputtong, P. (2006). Blurring Boundaries in Qualitative Health Research on Sensitive Topics. Qualitative Health Research, 16, 853-871. https://doi.org/10.1177/1049732306287526

Dickson-Swift, V., James, E. L., Kippen, S., \& Liamputtong, P. (2008). Risk to Researchers in Qualitative Research on Sensitive Topics: Issues and Strategies. Qualitative Health Research, 18, 133-144. https://doi.org/10.1177/1049732307309007

Dickson-Swift, V., James, E. L., Kippen, S., \& Liamputtong, P. (2009). Researching Sensitive Topics: Qualitative Research as Emotion Work. Qualitative Research, 9, 61-79. https://doi.org/10.1177/1468794108098031

Duncan, P., \& Stephenson, A. (2013). Participant Responsibility, Researcher Vulnerability and Empirical Ethics: A Reply to Loughlin. Journal of Evaluation in Clinical Practice, 19, 553-558. https://doi.org/10.1111/jep.12056

Emerald, E., \& Carpenter, L. (2015). Vulnerability and Emotions in Research: Risks, Dilemmas, and Doubts. Qualitative Inquiry, 21, 741-750. https://doi.org/10.1177/1077800414566688

Etherington, K. (2007). Working with Traumatic Stories: From Transcriber to Witness. International Journal of Social Research Methodology, 10, 85-97. https://doi.org/10.1080/13645570701334001

Hernández, M. G., Nguyen, J., Casanova, S., Suárez-Orozco, C., \& Saetermoe, C. L. (2013). Doing No Harm and Getting It Right: Guidelines for Ethical Research with Immigrant Communities. New Directions for Child \& Adolescent Development, 2013, 43-60. https://doi.org/10.1002/cad.20042

Horn, L. (2007). Research Vulnerability: An Illustrative Case Study from the South African Mining Industry. Developing World Bioethics, 7, 119-127.

https://doi.org/10.1111/dewb 151.x

Horstman, M., Aldiss, S., Richardson, A., \& Gibson, F. (2008). Methodological Issues When Using the Draw and Write Technique with Children Aged 6 to 12 Years. Qualitative Health Research, 18, 1001-1011. https://doi.org/10.1177/1049732308318230

Ismail, M. M., Gerrish, K., Naisby, A., Salway, S., \& Chowbey, P. (2014). Engaging Minorities in Researching Sensitive Health Topics by Using a Participatory Approach. 
Nurse Researcher, 22, 44-48. https://doi.org/10.7748/nr.22.2.44.e1268

Kaplowitz, M. D. (2000). Statistical Analysis of Sensitive Topics in Group and Individual Interviews. Quality and Quantity, 34, 419-431. https://doi.org/10.1023/A:1004844425448

Kästner, B., Behre, S., Lutz, N., Bürger, F., Luntz, S., Hinderhofer, K., Bendszus, M., Hoffmann, G. F., \& Ries, M. (2015). Clinical Research in Vulnerable Populations: Variability and Focus of Institutional Review Boards' Responses. PLoS ONE, 10, e0135997. https://doi.org/10.1371/journal.pone.0135997

Kelleya, M. C., Brazg, T., Wilfond, B. S., Lengua, L. J., Rivine, B. E., Martin-Herz, S. P., \& Diekema, D. S. (2016). Ethical Challenges in Research with Orphans and Vulnerable Children: A Qualitative Study of Researcher Experiences. International Health, 8, 187-196. https://doi.org/10.1093/inthealth/ihw020

Kia-Keating, M., Santacrose, D., \& Liu, S. (2017). Photography and Social Media Use in a Community-Based Participatory Research with Youth: Ethical Considerations. American Journal of Community Psychology, 60, 375-384. https://doi.org/10.1002/ajcp.12189

Kipnis, K. (2001). Vulnerability in Research Subjects: A Bioethical Taxonomy. Commissioned Paper at the University of Hawaii at Manoa.

http://www.aapcho.org/wp/wp-content/uploads/2012/02/Kipnis-VulnerabilityinResear chSubjects.pdf

Lajoie, C., Poleksic, J., Bracken-Roche, D., MacDonald, M. E., \& Racine, E. (2020). The Concept of Vulnerability in Mental Health Research: A Mixed Methods Study on Researcher Perspectives. Journal of Empirical Research on Human Research Ethics, 15, 128-142. https://doi.org/10.1177/1556264620902657

Lambrechts, D. (2014). Doing Research on Sensitive Topics in Political Science: Studying Organised Criminal Groups in Cape Town. Politikon: South African Journal of Political Studies, 41, 249-265. https://doi.org/10.1080/02589346.2014.905257

Leigh, J. (2015). Crossing the Divide between Them and Us: Using Photography to Explore the Impact Organisational Space Can Have on Identity and Child Protection Practice. Qualitative Social Work, 14, 416-435.

https://doi.org/10.1177/1473325014555442

Matsudaira, T. (2003). Cultural Influences on the Use of Social Support by Chinese Immigrants in Japan: "Face" as a Keyword. Qualitative Health Research, 13, 343-357. https://doi.org/10.1177/1049732302250130

Merry, L., Clausen, C., Gagnon, A. J., Carnevale, F., Jeannotte, J., Saucier, J.-F., \& Oxman-Martinez, J. (2011). Improving Qualitative Interviews with Newly Arrived Migrant Women. Qualitative Health Research, 21, 976-986. https://doi.org/10.1177/1049732311403497

Mitchell, W., \& Irvine, A. (2008). I'm Okay, You're Okay? Reflections on the Well-Being and Ethical Requirements of Researchers and Research Participants in Conducting Qualitative Fieldwork Interviews. International Journal of Qualitative Methods, 7, 31-44. https://doi.org/10.1177/160940690800700403

Muller, A. E., \& Gubrium, E. (2016). Researcher Linguistic Vulnerability. Qualitative Health Research, 26, 141-144. https://doi.org/10.1177/1049732315613312

Noue, G. R., \& Bush, A. (2010). Institution Review Board Rules: Should One Size Fit All Discipline? International Journal of Interdisciplinary Social Sciences, 5, 239-258. https://doi.org/10.18848/1833-1882/CGP/v05i08/51833

Parker, N., \& O’Reilly, M. (2013). "We Are Alone in the House”: A Case Study Addressing Researcher Safety and Risk. Qualitative Research in Psychology, 10, 341-354. https://doi.org/10.1080/14780887.2011.647261 
Peterson, P., Sackey, D., Kay, M., Correa-Velez, I., \& Nicholson, C. (2017). Using a Peer Led Researcher Model to Connect with Vulnerable Communities around Health. International Journal of Integrated Care, 17, 116-117. https://doi.org/10.5334/ijic.3177

Ryan, J. E., Smeltzer, S. C., \& Sharts-Hopko, N. C. (2019). Challenges to Studying Illicit Drug Users. Journal of Nursing Scholarship, 51, 480-488. https://doi.org/10.1111/jnu.12486

Snoek, A., \& Horstkötter, D. (2018). Ethical Issues in Research on Substance-Dependent Parents: The Risk of Implicit Normative Judgments by Researchers. Bioethics, 32, 620627. https://doi.org/10.1111/bioe.12514

Taylor, S. (2019). The Long Shadows Cast by the Field: Violence, Trauma, and the Ethnographic Researcher. Fennia, 197, 183-199. https://doi.org/10.11143/fennia.84792

von Benzon, N., \& van Blerk, L. (2017). Research Relationships and Responsibilities: “Doing” Research with "Vulnerable" Participants. Social \& Cultural Geography, 18, 895-905. https://doi.org/10.1080/14649365.2017.1346199

Wakai, S., Shelton, D., Trestman, R. L., \& Kesten, K. (2009). Conducting Research in Corrections: Challenges and Solutions. Behavioral Sciences \& the Law, 27, 743-752. https://doi.org/10.1002/bsl.894

Wilson, E., Kenny, A., \& Dickson-Swift, V. (2018). Ethical Challenges of Community Based Participatory Research: Exploring Researchers' Experience. International Journal of Social Research Methodology, 21, 7-24.

https://doi.org/10.1080/13645579.2017.1296714

Yiu, T. S. (2015). Methodological Reflections on Researching Lesbian, Gay, Bisexual and Transgender University Students in Hong Kong: To What Extent Are They Vulnerable Interview Subjects? Higher Education Research \& Development, 34, 722. https://doi.org/10.1080/07294360.2015.1051009

Yoshikawa, H., Whipps, M. D. M., \& Rojas, N. M. (2017). Commentary: New Directions in Developmentally Informed Intervention Research for Vulnerable Populations. Child Development, 88, 459-465. https://doi.org/10.1111/cdev.12736 